OUTP-98-13P

hep-th/9802146

\title{
Composite Operator Effective Potential Approach to $\mathrm{QED}_{3}$
}

\author{
A. Campbell-Smith \\ University Of Oxford, Department of Physics, Theoretical Physics, 1 Keble Road, \\ OXFORD, OX1 3NP, U.K.
}

\begin{abstract}
The composite operator effective potential is compared with the conventional Dyson-Schwinger method as a calculational tool for $(2+1)$-dimensional quantum electrodynamics. It is found that when the fermion propagator ansatz is put directly into the effective potential, it reproduces exactly the usual gap equations derived in the Dyson-Schwinger approach.
\end{abstract}




\section{Introduction.}

Studies of $N$-flavour Quantum Electrodynamics in three dimensions $\left(\mathrm{QED}_{3}\right)$ have long been made in order to learn about dynamical mass generation (and hence chiral symmetry breaking) in a relatively simple context [1, 2, 3, 4. Initially the hope was to gain insight into dynamical symmetry breaking in other asymptotically free theories, in particular Yang-Mills theories in four dimensions. Subsequently there has been interest in $\mathrm{QED}_{3}$ as a model for high- $\mathrm{T}_{\mathrm{c}}$ superconductors [5]. Here the broken, massive phase of $\mathrm{QED}_{3}$ gives a mechanism for spontaneously breaking the $U(1)$ gauge symmetry which is necessary for superconductivity, and deviations from the trivial infra red fixed point could be responsible for the observed non-Fermi liquid behaviour in the normal phase of these materials [6].

Considering $N$ fermion flavours allows one to perform a large $N$ expansion and keep only dominant terms in this limit. This is a convenient calculational tool for it admits a form for the full gauge boson propagator (namely that with massless fermion loops summed [1]) without requiring that the Dyson-Schwinger equation for the propagator be solved. There is a further significance of working with $N$ flavours in the possibility that there exists a critical number of flavours [2, 7] above which no symmetry breaking occurs. Much of the evidence for the existence of a critical flavour number lies in numerical work and the analytical studies that have been made require great simplifications to be tractable; indeed there is evidence for no critical behaviour in the flavour number (see e.g. 狛).

Non-perturbative treatments of $\mathrm{QED}_{3}$ are normally based on solving systems of Dyson-Schwinger equations. To make the systems soluble the equations for the gauge boson self energy are dropped in favour of the propagator resummed in large $N$; the equations for the vertex are replaced by an ansatz discussed further below. This leaves only the Dyson-Schwinger equation for the fermion propagator to be solved; the approach taken is to substitute a (rather general) ansatz for the propagator into the equations and then solve for the functions appearing in the ansatz.

The system of Dyson-Schwinger equations for a theory can in principle be derived from a composite operator effective action [8] via a well-defined minimization technique. It is the purpose of this paper to consider whether the same physics is found if one puts the ansatz directly into the effective action. The same minimization technique can then be used to derive the "gap equations" for the functions in the ansatz directly from the effective action, and these can then be compared with the conventional Dyson-Schwinger results. The system of Dyson-Schwinger equations used is heavily truncated and the effective action is computed to two loop level only; it is therefore not immediately obvious that the results of the two computations will coincide. Indeed in general the calculations may give different answers (e.g. 91), and comparing the methods can allow one to estimate the limitations of the approximations used. Since the approximations described above are used almost universally in work on $\mathrm{QED}_{3}$ it is important to compare the two methods within the those limits. It will be shown that the composite operator effective action method gives exactly the usual (Dyson-Schwinger) gap equations, after simplification of the "raw" equations which come directly from the minimization procedure. The amount of computational effort required is rather surprising, and may have important consequences for future work at finite temperature; it is probable that finite temperature Dyson-Schwinger approaches would be more economical.

This paper is organized as follows: in section 2, the basic formalism for composite operator effective actions is reviewed, rewritten for fermionic fields; section 3 contains a comparison of the effective action and Dyson-Schwinger equation approaches and in section 4 are the details of the effective action calculation. Conclusions are appended to these sections. 


\section{The Composite Operator Effective Action.}

The composite operator effective action [8] is a generalization of the conventional (quantum) effective action; for fermionic fields it depends not only on $\psi(x)$ and $\bar{\psi}(x)$ - possible expectation values of the quantum fields $\Psi(x)$ and $\bar{\Psi}(x)$ - but also on $S(x, y)$, a possible expectation value of the time ordered product $T\{\bar{\Psi}(x) \Psi(y)\}$. Physical solutions require that the effective action be stationary with respect to variations in the expectation values:

$$
\begin{aligned}
& \frac{\delta \Gamma[\psi, \bar{\psi}, S]}{\delta \psi(x)}=0, \\
& \frac{\delta \Gamma[\psi, \bar{\psi}, S]}{\delta \bar{\psi}(x)}=0, \\
& \frac{\delta \Gamma[\psi, \bar{\psi}, S]}{\delta S(x, y)}=0 .
\end{aligned}
$$

For this reason the formalism is especially suitable for studying dynamical symmetry breaking, for although the first two of equations (1) may only have the symmetric solution $\psi(x)=\bar{\psi}(x)=0$, symmetry breaking solutions may exist for the last equation.

First we define the generating functional $Z[\eta, \bar{\eta}, \Lambda]\left(\int_{x}=\int d^{3} x\right)$ :

$$
\begin{aligned}
Z[\eta, \bar{\eta}, \Lambda] & =\exp \{i W[\eta, \bar{\eta}, \Lambda]\} \\
& =\int D \Psi D \bar{\Psi} \exp \left\{i \left[I[\Psi, \bar{\Psi}]+\int_{x}(\bar{\eta}(x) \Psi(x)+\bar{\Psi}(x) \eta(x))\right.\right. \\
& \left.\left.+\int_{x} \int_{y} \bar{\Psi}(x) \Lambda(x, y) \Psi(y)\right]\right\},
\end{aligned}
$$

where $\{\eta, \bar{\eta}\}$ and $\Lambda$ are fermionic and bosonic source currents respectively. The $\Psi$

and $\bar{\Psi}$ integrations are functional, and $I[\Psi, \bar{\Psi}]$ is the classical effective action. The composite operator effective action is the double Legendre transform of $W[\eta, \bar{\eta}, \Lambda]$. Defining

$$
\begin{aligned}
\frac{\delta W[\eta, \bar{\eta}, \Lambda]}{\delta \eta(x)} & =-\bar{\psi}(x), \\
\frac{\delta W[\eta, \bar{\eta}, \Lambda]}{\delta \bar{\eta}(x)} & =\psi(x), \\
\frac{\delta W[\eta, \bar{\eta}, \Lambda]}{\delta \Lambda(x, y)} & =\bar{\psi}(x) \psi(y)+S(x, y),
\end{aligned}
$$

one can eliminate $\eta, \bar{\eta}$ and $\Lambda$ in favour of $\psi, \bar{\psi}$ and $S$ in the Legendre transform:

$$
\begin{aligned}
\Gamma[\psi, \bar{\psi}, S] & =W[\eta, \bar{\eta}, \Lambda]-\int_{x}(\bar{\psi}(x) \eta(x)+\bar{\eta}(x) \psi(x)) \\
& -\int_{x} \int_{y} \bar{\psi}(x) \Lambda(x, y) \psi(y)-\int_{x} \int_{y} S(x, y) \Lambda(y, x) .
\end{aligned}
$$

It is then easy to show that

$$
\begin{aligned}
\frac{\delta \Gamma[\psi, \bar{\psi}, S]}{\delta \psi(x)} & =\bar{\eta}(x)+\int_{y} \bar{\psi}(y) \Lambda(y, x), \\
\frac{\delta \Gamma[\psi, \bar{\psi}, S]}{\delta \bar{\psi}(x)} & =-\eta(x)-\int_{y} \Lambda(x, y) \psi(y), \\
\frac{\delta \Gamma[\psi, \bar{\psi}, S]}{\delta S(x, y)} & =-\Lambda(x, y) .
\end{aligned}
$$


Physical processes correspond to vanishing sources, so equations (5) provide a derivation of the stationary requirements, equations (11).

Since we will be interested only in translation-invariant solutions we can set $\psi$ and $\bar{\psi}$ to constants and take $S(x, y)$ to be a function only of the separation $x-y$. This leads to a generalization of the effective potential [8], defined by

$$
V[\psi, \bar{\psi}, S] \int_{x}=-\Gamma[\psi, \bar{\psi}, S] .
$$

Then a series expansion for $V[\psi, \bar{\psi}, S]$ is relatively easy to find [8]; the specific expansion for $\mathrm{QED}_{3}$ is given in section 3 .

Composite operator effective actions depending on possible expectation values of irreducible three-point, four-point etc. functions can be defined simply by taking further Legendre transforms, however the "bilocal" effective action defined above is sufficient to study $\mathrm{QED}_{3}$.

\section{The Effective Potential and Dyson-Schwinger Equations in $\mathrm{QED}_{3}$.}

The Lagrangian density for $N$-flavour $\mathrm{QED}_{3}$ is as follows:

$$
\mathcal{L}=-\frac{1}{4} F_{\mu \nu}^{2}+i \sum_{k=1}^{N} \bar{\Psi}_{k} \gamma_{\mu} \mathcal{D}_{\mu} \Psi_{k}+\mathcal{L}_{\mathrm{GF}}
$$

where $F_{\mu \nu}$ and $\mathcal{D}_{\mu}$ are the usual $U(1)$ curvature tensor and covariant derivative respectively; the fermions are represented by four-component Dirac spinors $\Psi_{k}$ so that the theory has a chiral symmetry and can exhibit mass generation [2]. The gauge fixing term is not written explicitly: throughout the calculation we shall work in Landau gauge. The series expansion for the composite operator effective potential in momentum space is then [B] $\left(\int_{k}=\int d^{3} k /(2 \pi)^{3}\right)$ :

$$
\begin{aligned}
V[S, D] & =i N \int_{p} \operatorname{tr}\left\{\ln \left[S^{-1}(p) S_{0}(p)\right]+S_{0}^{-1}(p) S(p)-1\right\} \\
& -\frac{i}{2} \int_{p} \operatorname{tr}\left\{\ln \left[D^{-1}(p) D_{0}(p)\right]+D_{0}^{-1}(p) D(p)-1\right\} \\
& +V_{2}[S, D] .
\end{aligned}
$$

The functions $S$ and $D$ are candidate full non-perturbative propagators for the fermions and gauge fields respectively, and the subscripts 0 denote their bare counterparts. The effective potential is minimized with respect to (functional) variations in $S$ and $D$ to determine the physical non-perturbative propagators for the theory, as described in section 2. $-V_{2}[S, D]$ is the sum of all two-particle irreducible vacuum graphs with the propagators set equal to $S$ and $D$ and with bare (not dressed) vertices. To consider more general vertices one would have to got to a "trilocal" effective action (i.e. three Legendre transforms), and require that this new object be stationary also with respect to variations in a vertex ansatz.

The graphs contributing to $V_{2}[S, D]$ up to three loop level are depicted in figure 1. There are also two-particle reducible graphs which would have to be considered in an ordinary (one-particle irreducible) effective action calculation but which do not contribute to $V_{2}[S, D]$; these are depicted up to three loop level in figure 2.

We will truncate the series at the two loop level, as this will be sufficient for comparison with the usual Dyson-Schwinger method for $\mathrm{QED}_{3}$. The two-loop term 


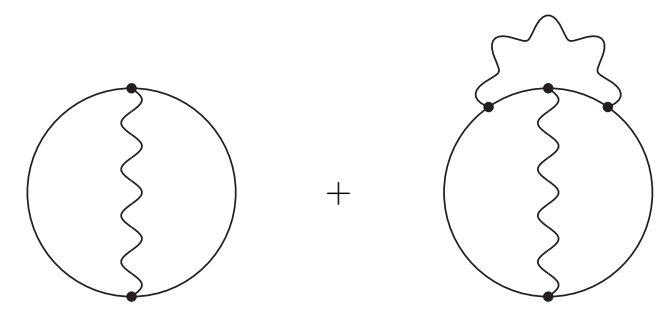

Figure 1: Two-particle irreducible graphs contributing to $V_{2}$, up to three loop level.

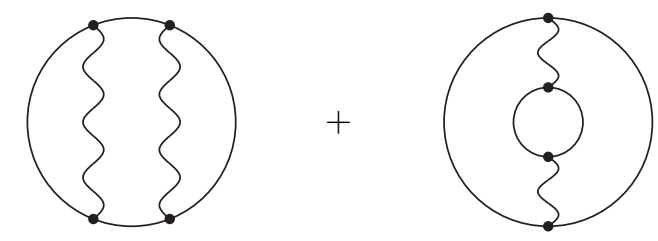

Figure 2: Two-particle reducible graphs not contributing to $V_{2}$ (to three loop level), but which would contribute to the one-particle irreducible effective action.

in $V_{2}$ follows:

$$
V_{2}[S, D]=\frac{e^{2} N}{2} \int_{p} \int_{k} \operatorname{tr}\left\{\gamma_{\mu} S(p) \Gamma_{\nu} S(p+k) D_{\mu \nu}(k)\right\} .
$$

For the purposes of comparison with the Dyson-Schwinger method we keep the "general" vertex $\Gamma$; when we turn to the effective potential calculation proper in section 4 this will be reset to the bare vertex. On demanding that $V[S, D]$ be stationary with respect to variations in $S$ and $D$ the usual Dyson-Schwinger equations are obtained:

$$
\begin{gathered}
S^{-1}(p)=S_{0}^{-1}(p)+e^{2} \int_{k}\left\{S(k) \gamma_{\mu} D_{\mu \nu}(q-k) \Gamma_{\nu}\right\} \\
D_{\mu \nu}^{-1}(p)=D_{0}^{-1}{ }_{\mu \nu}(p)-e^{2} N \int_{k}\left\{\gamma_{\mu} S(k) \Gamma_{\nu} S(k+p)\right\} .
\end{gathered}
$$

Now these equations can be solved for $S$ and $D$ (with some ansatz or a further Dyson-Schwinger equation for the vertex) to give the full non-perturbative propagators which minimize the effective potential. Since the interest is in possible dynamical mass generation in the fermion sector we use an ansatz for the fermion propagator and solve for the functions appearing therein:

$$
S^{-1}(p)=-i(A(p) \not p+B(p)) .
$$

Here $A$ and $B$ are both assumed to be scalar functions, so that this ansatz is rather general in that it only omits possible parity-breaking mass terms.

The gauge propagator is parameterized by the vacuum polarization, and we approximate this function by the leading order truncation in $1 / N$ [1]:

$$
D_{\mu \nu}(p)=-\frac{i}{p^{2}} \frac{1}{1+\alpha / p}\left(\delta_{\mu \nu}-\frac{p_{\mu} p_{\nu}}{p^{2}}\right) ;
$$




$$
\alpha=\frac{e^{2} N}{8} .
$$

The only requirement put on the vertex function is that it is dependent on the magnitudes alone of the momenta at the vertex, but it must also be consistent with the Ward-Takahashi identity 10]:

$$
\Gamma_{\mu}(p, k)=-i \gamma_{\mu} G\left(p^{2}, k^{2}\right) .
$$

The full vertex ansatz (14) appears only once in $V_{2}$ to avoid double-counting. This treatment of the vertex is consistent with that in Dyson-Schwinger calculations; when we turn to the effective potential calculation, the function $G$ will be set to unity. In order to take account of higher loop effects in the vertex, one has to consider the "trilocal" effective action discussed briefly above.

When these functions are substituted into the Dyson-Schwinger equations (10, 11) the following gap equations for $A$ and $B$ can be derived:

$$
\begin{aligned}
& A(q)=1-\frac{\alpha}{\pi^{2} N} \frac{1}{q^{3}} \int_{0}^{\infty} d k \frac{k A(k) G\left(q^{2}, k^{2}\right)}{\mathcal{K}(k)} \mathcal{I}_{1}(k, q ; \alpha), \\
& B(q)=\frac{\alpha}{\pi^{2} N} \frac{1}{q} \int_{0}^{\infty} d k \frac{k B(k) G\left(q^{2}, k^{2}\right)}{\mathcal{K}(k)} \mathcal{I}_{2}(k, q ; \alpha) ;
\end{aligned}
$$

with the integrals given by:

$$
\begin{aligned}
\mathcal{I}_{1}(k, q ; \alpha) & =\alpha^{2} \ln \left[\frac{k+q+\alpha}{|k-q|+\alpha}\right]-\alpha(k+q-|k-q|) \\
& -\frac{1}{\alpha}\left|k^{2}-q^{2}\right|(k+q-|k-q|)+2 k q \\
& -\frac{1}{\alpha^{2}}\left(k^{2}-q^{2}\right)\left\{\ln \left[\frac{k+q+\alpha}{|k-q|+\alpha}\right]-\ln \left[\frac{k+q}{k-q}\right]\right\} ; \\
\mathcal{I}_{2}(k, q ; \alpha) & =4 \ln \left[\frac{k+q+\alpha}{|k-q|+\alpha}\right],
\end{aligned}
$$

and

$$
\mathcal{K}(k)=A^{2}(k) k^{2}+B^{2}(k) .
$$

These equations can now be solved (using analytical and numerical methods) for $A$ and $B$ to yield the full fermion propagator which minimizes the effective potential.

The approach taken in this paper will differ from the conventional DysonSchwinger method outlined above. We shall use the ansatz (12) directly in the effective potential, equation (8), and demand that $V[A, B]$ be stationary with respect to (functional) variations in $A$ and $B$. We can then derive the "gap equations" for $A$ and $B$ and compare them with the equations found in the Dyson-Schwinger approach, equations (15).

\section{Gap Equations from the Effective Potential.}

The effective potential comprises three natural parts; the trace over the fermion propagator terms, the trace over the gauge boson propagator terms, and the sum of two-particle irreducible vacuum graphs. The second of these will play no part in the variation to be described: we will again use the leading order (in $1 / N$ ) truncation for the gauge propagator (13). After substituting (12), the variation of the pure fermion part is given by:

$$
\begin{aligned}
\frac{\delta V_{f}[A, B]}{\delta A(q)} & =\frac{4 N i}{(2 \pi)^{3}} \frac{A^{2}(q) q^{4}(A(q)-1)+B^{2}(q) q^{2}(A(q)+1)}{\mathcal{K}^{2}(q)} \\
\frac{\delta V_{f}[A, B]}{\delta B(q)} & =\frac{4 N i}{(2 \pi)^{3}} \frac{A(q) B(q) q^{2}(A(q)-2)+B^{3}(q)}{\mathcal{K}^{2}(q)}
\end{aligned}
$$


When these variations alone are set to vanish, the equations have the solutions $A=1, B=0$, consistent with the free propagators. The variation of the loop part is:

$$
\begin{aligned}
\frac{\delta V_{2}[A, B]}{\delta A(q)}= & -\frac{i e^{2} N}{2 \pi^{3}} \frac{2 A(q) B(q) q^{2}}{\mathcal{K}^{2}(q)} \int_{k} \frac{B(k)}{\mathcal{K}(k)} \frac{2}{(k-q)^{2}} \frac{1}{1+\alpha /|k-q|} \\
& -\frac{i e^{2} N}{2 \pi^{3}}\left[\frac{2 A^{2}(q) q^{2}}{\mathcal{K}^{2}(q)}-\frac{1}{\mathcal{K}(q)}\right] \int_{k} \frac{A(k)}{\mathcal{K}(k)} \frac{2}{(k-q)^{4}} \frac{q \cdot(k-q) k \cdot(k-q)}{1+\alpha /|k-q|} \\
\frac{\delta V_{2}[A, B]}{\delta B(q)}= & -\frac{i e^{2} N}{2 \pi^{3}}\left[\frac{2 B^{2}(q)}{\mathcal{K}^{2}(q)}-\frac{1}{\mathcal{K}(q)}\right] \int_{k} \frac{B(k)}{\mathcal{K}(k)} \frac{2}{(k-q)^{2}} \frac{1}{1+\alpha /|k-q|} \\
& -\frac{i e^{2} N}{2 \pi^{3}} \frac{2 A(q) B(q)}{\mathcal{K}^{2}(q)} \int_{k} \frac{A(k)}{\mathcal{K}(k)} \frac{2}{(k-q)^{4}} \frac{q \cdot(k-q) k \cdot(k-q)}{1+\alpha /|k-q|}
\end{aligned}
$$

Computing the resulting angular integrations, performing some simplifications, and setting the variations to vanish we obtain the "raw" gap equations:

$$
\begin{aligned}
0 & =A^{2}(q) q^{4}(A(q)-1)+B^{2}(q) q^{2}(A(q)+1)-\frac{2 \alpha}{N \pi^{2}} 2 A(q) B(q) q^{2} \int d k \mathcal{J}_{1}(k, q ; \alpha) \\
& -\frac{2 \alpha}{N \pi^{2}}\left(A^{2}(q) q^{2}-B^{2}(q)\right) \int d k \mathcal{J}_{2}(k, q ; \alpha) \\
0 & =A(q) B(q) q^{2}(A(q)-2)+B^{3}(q)+\frac{2 \alpha}{N \pi^{2}}\left(A^{2}(q) q^{2}-B^{2}(q)\right) \int d k \mathcal{J}_{1}(k, q ; \alpha) \\
& -\frac{2 \alpha}{N \pi^{2}} 2 A(q) B(q) \int d k \mathcal{J}_{2}(k, q ; \alpha)
\end{aligned}
$$

where the integrals are given by:

$$
\begin{aligned}
\mathcal{J}_{1}(k, q, \alpha) & =k^{2} \frac{B(k)}{\mathcal{K}(k)} \frac{2}{k q} \ln \left[\frac{k+q+\alpha}{|k-q|+\alpha}\right] \\
\mathcal{J}_{2}(k, q ; \alpha) & =2 k^{2} \frac{A(k)}{\mathcal{K}(k)}\left(\frac{\left(k^{2}-q^{2}\right)^{2}-\alpha^{4}}{4 k q \alpha^{2}} \ln \left[\frac{k+q+\alpha}{|k-q|+\alpha}\right]\right. \\
& -\frac{\left(k^{2}-q^{2}\right)^{2}}{4 k q \alpha^{2}} \ln \left[\frac{k+q}{|k-q|}\right]+\frac{\alpha}{4 k q}[k+q-|k-q|]-\frac{1}{2} \\
& \left.+\frac{\left(k^{2}-q^{2}\right)^{2}}{4 k q \alpha}\left[\frac{1}{|k-q|}-\frac{1}{k+q}\right]\right) .
\end{aligned}
$$

Since the momentum integrals appearing in equations $(20,21)$ are the same we can eliminate each of them in turn: after a little simplification this yields the usual gap equations obtained from the Dyson-Schwinger approach, equations (15), up to the replacement $G \rightarrow 1$ which is imposed by considering only a "bilocal" effective action.

\section{Concluding Remarks.}

The fact that the composite operator effective potential yields the same gap equations (15) whether the ansätze are put in before or after the functional variation demonstrates explicitly that it is equivalent to the Dyson-Schwinger method for $\mathrm{QED}_{3}$. It is by no means obvious from the "raw" gap equations (20, 21) that they will reduce on simplification to the normal Dyson-Schwinger results; since the effective potential and Dyson-Schwinger methods are frequently applied to $\mathrm{QED}_{3}$ it is 
important to have checked their equivalence within the framework of the approximations normally used. The consistency here is a basis for confidence that the ansätze used are sufficiently consistent and general: in other work [9] it has been found that overly restrictive ansätze lead to differences between Dyson-Schwinger type approaches and the results of composite operator effective action computations.

To better model high- $T_{c}$ superconductivity, finite temperature effects should really be included. The conclusion that can be drawn from the present calculation is that using the composite operator effective potential for such work (see e.g. [11]) is not likely to yield additional information nor to simplify the computation compared with a finite temperature Dyson-Schwinger method.

At both finite and zero temperature the choice of vertex function remains the major problem with this sort of calculation: how to make an ansatz that is consistent with the Ward-Takahashi identity, when that identity requires the full fermion propagator. This problem is not resolved by using the "bilocal" composite operator effective potential; however, in principle this problem could be solved completely by using a "trilocal" composite operator effective action, leading to a prediction for the vertex. However the calculation would be extremely difficult. The most promising way to attack this particular problem is by using the non-local gauge [7], in which the wave-function renormalization $A$ is forced to be unity. This approach

does not work well within the composite operator effective potential formalism, for the resulting analogue of equation (21) for $B$ is intractable.

\section{Acknowledgments.}

The author would like to thank G. Amelino-Camelia for suggesting the problem, and to thank I Aitchison, G. Amelino-Camelia and N. Mavromatos for helpful and entertaining discussions. Thanks are also due to P.P.A.R.C. (U.K.) for a research studentship (number 96314661).

\section{References}

[1] R. Pisarski; Physical Review D 29, 2423 (1984).

[2] T. Appelquist, M. Bowick, D. Karabali, and L. Wijewardhana; Physical Review D 33, $3704(1986)$.

[3] T. Appelquist, D. Nash and L. Wijewardhana; Physical Review Letters 60, 2575 (1988).

[4] M. Pennington and D. Walsh; Physics Letters B 253, 246 (1991).

[5] N. Dorey and N. Mavromatos; Physics Letters B 250, 107 (1990).

N. Dorey and N. Mavromatos; Nuclear Physics B 386, 614 (1992).

[6] I. Aitchison and N. Mavromatos; Physical Review B 53, 9321 (1996).

I. Aitchison, G. Amelino-Camelia, M. Klein-Kreisler, N. Mavromatos and D. McNeill; Physical Review B 56, 2836 (1997).

[7] K.-I. Kondo, T. Ebihara, T Iizuka and E. Tanaka; Nuclear Physics B 434, 85 (1995).

P. Maris; Physical Review D 54, 4049 (1996).

I. Aitchison, N. Mavromatos and D. McNeill; Physics Letters B 402, 154 (1997).

[8] J.M. Cornwall, R. Jackiw and E. Tomboulis; Physical Review D 10, 2428 (1974). 
[9] G. Amelino-Camelia; Physical Review D 49, 2740 (1994).

[10] P. Maris; Physical Review D 54, 4049 (1996).

[11] G. Amelino-Camelia and S.-Y. Pi; Physical Review D 47, 2356 (1993). 